\title{
lonospheric ion upwelling in the wake of flux transfer events at the dayside magnetopause
}

Article

Published Version

Lockwood, M., Smith, M. F., Farrugia, C. J. and Siscoe, G. L. (1988) lonospheric ion upwelling in the wake of flux transfer events at the dayside magnetopause. Journal of Geophysical Research, 93 (A6). pp. 5641-5654. ISSN 0148-0227 doi: https://doi.org/10.1029/JA093iA06p05641 Available at https://centaur.reading.ac.uk/38894/

It is advisable to refer to the publisher's version if you intend to cite from the work. See Guidance on citing.

Published version at: http://dx.doi.org/10.1029/JA093iA06p05641

To link to this article DOI: http://dx.doi.org/10.1029/JA093iA06p05641

Publisher: American Geophysical Union

All outputs in CentAUR are protected by Intellectual Property Rights law, including copyright law. Copyright and IPR is retained by the creators or other copyright holders. Terms and conditions for use of this material are defined in the End User Agreement.

www.reading.ac.uk/centaur 
Central Archive at the University of Reading

Reading's research outputs online 
Abstract. The effects of flux transfer events (FTE) on the dayside auroral ionosphere are studied, using a simple twin-vortex model of induced ionospheric plasma flow. It is shown that the predicted and observed velocities of these flows are sufficient to drive nonthermal plasma in the $F$ region, not only within the newly opened flux tube of the FTE, but also on the closed, or "old" open, field lines around it. In fact, with the expected poleward neutral wind, the plasma is more highly nonthermal on the flanks of, but outside, the open flux tube: EISCAT observations indicate that plasma is indeed driven into nonthermal distributions in these regions. The nonthermal plasma is thereby subject to additional upforce due to the resulting ion temperature anisotropy and transient expansion due to Joule heating and also to ion accelerations associated with the FTE field aligned current system. Any upflows produced on closed field lines in the vicinity of the FTE are effectively bunched-up in the "wake" of the FTE. Observations from the AMPTE-UKS satellite at the magnetopause reveal ion upflows of energy $\sim 100 \mathrm{eV}$ flowing out from the ionosphere on closed field lines which are only found in the wake of the FTE. Such flows are also only found shortly after two, out of all the FTEs observed by AMPTEUKS. The outflow from the ionosphere is two orders of magnitude greater than predicted for the "classical" polar wind. It is shown that such ionospheric ion flows are only expected in association with FTEs on the magnetopause which are well removed from the sub-solar point-either towards dusk or, as in the UKS example discussed here, towards dawn. It is suggested that such ionospheric ions will only be observed if the center of the FTE open flux tube passes very close to the satellite. Consequently, we conclude the ion upflows presented here are probably driven by the second of two possible source FTEs and are observed at the satellite with a laq

\footnotetext{
${ }^{1}$ Rutherford Appleton Laboratory, Chilton, Didcot, Oxon, England.

2 Also Visiting Honorary Lecturer, Blackett Laboratory, Imperial College, London.

${ }^{3}$ Mullard Space Science Laboratory, Department of Physics and Astronomy, University College London.

${ }^{4}$ Presently at Southwest Research Institute, San Antonio, Texas 78284.

5 Blackett Laboratory, Imperial College, London.

${ }^{6}$ University of California at Los Angeles, Department of Atmospheric Sciences.
}

Copyright 1988 by the American Geophysical Union

Paper number 7 A9276

$0148-0227 / 88 / 007 A-9276 \$ 05.00$ after the FTE which is less than their time-offlight.

\section{Introduction}

The phenomenon of short-lived ( $5 \mathrm{~min})$, spatially localized $\left(\sim 1 R_{E}\right)$ reconnection at the dayside magnetopause in a "flux transfer event" (FTE) was first identified in 1978 by Russell and Elphic. Recent ground-based observations by the STARE and EISCAT radars have found flow patterns consistent with twin vortices of spatial scales of several hundred kilometres, in the dayside auroral ionosphere [Goertz et al., 1985; Todd et al., 1986, 1988]. Such a flow pattern had been predicted as the ionospheric signature of FTEs [Cowley, 1984; Southwood, 1985, 1987, Southwood et al., 1988]. Subsequently, Lockwood et al., [1987, 1988] noted that the ion drifts within the twin-vortex are sufficient to drive the $F$ region plasma nonthermal and showed the EISCAT spectra could only be explained by the presence of nonMaxwellian ion velocity distributions. K. Suvanto et al. (The influence of non-Maxwellian and anisotropic bi-Maxwellian ion velocity distributions on ionospheric ion outflows into the magnetosphere, submitted to Journal of Geophysical Research, 1987) have shown that for the nonthermal plasma found within the twinvortex, there is a considerable upforce on the ions, due to the divergence of geomagnetic field lines with altitude, in addition to the transient upwelling due to any rise in parallel ion temperature. The transient ion upflows for isotropically, Joule-heated plasma were modelled by Gombosi and Killeen [1987].

Lockwood and Fuller-Rowell (1987a, b) predicted the occurrence of nonthermal $F$ region plasma due to the global pattern of plasma convection. In their simulations, the neutral wind was computed self-consistently, as was the temperature and composition of the neutral gas. The occurrence of nonthermal plasma was identified from the value of $\mathrm{D}^{\prime}$, the ratio of the velocity difference between the ions and neutrals to the neutral thermal speed. St.-Maurice and Schunk [1979] found the ion velocity distribution showed marked departures from a bi-Maxwellian if D' exceeded about 0.75 from theoretical computations using the relaxation model of ion-neutral collisions. A more realistic collisional model was employed by Barakat et al. [1983], who mixed resonant charge exchange and polarization elastic scatter in their Monte Carlo simulations. Their results showed that the distribution function given by the relaxation model is quite a good approximation, but that the $D^{\prime}$ required to drive it is a little larger than the simpler model predicts. The threshold for nonthermal effects is $D^{\prime} \sim 1.5$, as the polarization scatter tends to destroy the nonthermal ion velocity distribution which the charge exchange collisions tend to build. It should also be noted that the Joule 
heating is proportional to the square of $\mathrm{D}^{\prime}$, so whether by the transient upwelling due to Joule heating (discussed by Gombosi and killeen [1987]) or by the "hydrodynamic mirror force" (discussed by Suvanto et al., 1987), $D^{\prime}$ is expected to be an indicator of plasma upwelling. Lockwood and Fuller-Rowell [1987a, b] noted that areas of high predicted $D^{\prime}$ are those where large and heavy (mass > $4 \mathrm{amu}$ ) suprathermal ion outflows from the ionosphere are indeed observed.

The effects of Joule heating and the hydrodynamic mirror force could give outward fluxes of ionospheric ions which exceed the values of the "classical" polar wind (see review by Lockwood [1986]), and which can contain heavy (mass > 4 amu) ions. However, their energy is not expected to greatly exceed the $\sim 0.3 \mathrm{eV}$ of the classical polar wind: time-dependent expansion gives ions of energy up to only a few ev [Schunk, 1986]. Hence additional processes are required to explain ionospheric ions of energies of hundreds of ev. A wide variety of such processes are expected on auroral field lines over a wide range of altitudes and have been reviewed by e.g., Moore [1986] and Schunk [1986]. Some lower altitude examples include parallel acceleration due to the high electron temperatures due to auroral precipitation [Barakat and Schunk, 1984]; acceleration by magnetohydrodynamic waves [Moore, 1986]; and resonance with electrostatic ion cyclotron waves driven by field-aligned currents [Dusenbery and Lyons, 1981], but there are many other possible mechanisms. Precipitation, magnetohydrodynamic waves and field-aligned currents are all present with high intensities in the vicinity of a flux transfer event. Hence it is reasonable to expect that, once released from the ionosphere, some ions will undergo acceleration to energies of hundreds of electron volts or more.

In section 2 we evaluate which regions in the vicinity of a flux transfer event are subject to F region plasma upwellings, and in section 3 consider the transport of plasma, both within and in the "wake" [Siscoe and Lockwood, 1986] of the open flux tube of the FTE. In section 4 we consider AMPTE-UKS observations of ionospheric ion outflows in the vicinity of an FTE, and in section 5 EISCAT observations of nonthermal plasma in the ionosphere in the vicinity of an apparent FTE signature.

\section{Predictions of $D^{\prime}$ in the vicinity of a Flux Transfer Event}

The calculations made here employ some input values taken from the steady state (in a diurnal sense) predictions of the coupled UCL/University of Sheffield three-dimensional, time-dependent numerical model of the coupled ionosphere-

thermosphere system, as presented by Lockwood and Fuller-Rowel1 [1987a, b]. In the "throat" region of the convection pattern, which maps to the dayside magnetopause (where flux transfer events occur) the predicted neutral temperature, $T_{n}$, is about $1100 \mathrm{~K}$ and the neutral wind is generally poleward and has a range of speeds of roughly 100-500 $\mathrm{m} \mathrm{s}^{-1}$ depending on location. These predictions are made for December solstice in the northern hemisphere (winter) cusp, with F10.7 = - 165, TIROS activity level of $7\left(\mathrm{~K}_{\mathrm{p}} \approx 3\right)$ and nega- tive $B_{y}$ component of the interplanetary magnetic field.

We make use of the definition of $D^{\prime}$ employed by Lockwood and Fuller-Rowell [1987a] and Farmer et al. [1988], namely,

$$
D^{\prime}=\frac{\left|\bar{v}_{i}-\bar{v}_{n}\right|}{v_{t h}}=\frac{\left|\bar{v}_{i}-\bar{v}_{n}\right|}{\sqrt{2 k T_{n} / m_{n}}}
$$

where $\bar{v}_{i}$ is the ion velocity, $\bar{v}_{n}$ the neutral velocity, $v_{t h}$ the two-dimensional (field perpendicular) neutral speed. For the conditions in the "throat" region given above, $v_{t h}$ is roughly 1 $\mathrm{km} \mathrm{s}^{-1}$, and we adopt that value in this paper. Reasonable variations of $v_{t h}$ about this value will cause variations in $D^{\prime}$, but not materially effect the conclusions drawn.

The model employed here for the ion flows, $v_{i}$, induced by a flux transfer event, assumes a circular cross section flux tube (often referred to as "the FTE") has been connected to the IMF. After one or more Alfuen wave bounce periods, a footprint of the flux tube in the ionosphere. also of circular cross section, is moved through the background plasma with velocity $\bar{\nabla}_{0}$ in the (unspecified) $x$ direction [Southwood, 1987]. The twin vortex flow pattern established by this is given by solution of Laplace's equation for an assumed incompressible ionosphere, and the flow is exactly analogous to the well-known fluid flow around a moving cylindrical object [Darwin, 1953; Milne-Thompson, 1955]: outside the open (connected to the IMF) flux tube, the velocity, $v_{i}$, is given by [Farrugia et al., 1987]:

$$
\begin{aligned}
& v_{r}=v_{0} \frac{a^{2}}{r^{2}} \cos \theta \\
& v_{\theta}=v_{0} \frac{a^{2}}{r^{2}} \sin \theta
\end{aligned}
$$

and inside it the flow is constant

$$
\begin{aligned}
& v_{r}=v_{o} \cos \theta \\
& v_{\theta}=-v_{o} \sin \theta
\end{aligned}
$$

Where $a$ is the radius of the open flux tube ( $a$ function of altitude) and $r$ and $\theta$ are polar coordinates defined in Figure 1a. The neutral wind velocity $\bar{v}_{n}$ makes an angle $\alpha$ with the $x$ direction.

$$
\begin{aligned}
& v_{n r}=v_{n} \cos (\theta-\alpha) \\
& v_{n \theta}=-v_{n} \sin (\theta-\alpha)
\end{aligned}
$$

Within the duration of an FTE the neutral wind is unaltered by the ion flows, hence $\bar{v}_{n}$ is taken as constant.

In Figure 1b a "snapshot" of the ion flow streamlines (or electric equipotentials) in a stationary frame is plotted, as described by equations (2) and (3), and shows the classic twin-vortex flow pattern, which we adopt as our model of ion flows associated with an FTE. Ionospheric flows have been observed by Todd et al. [1986] which are fully consistent with Figure 1b. This is a snapshot of the flows in this 


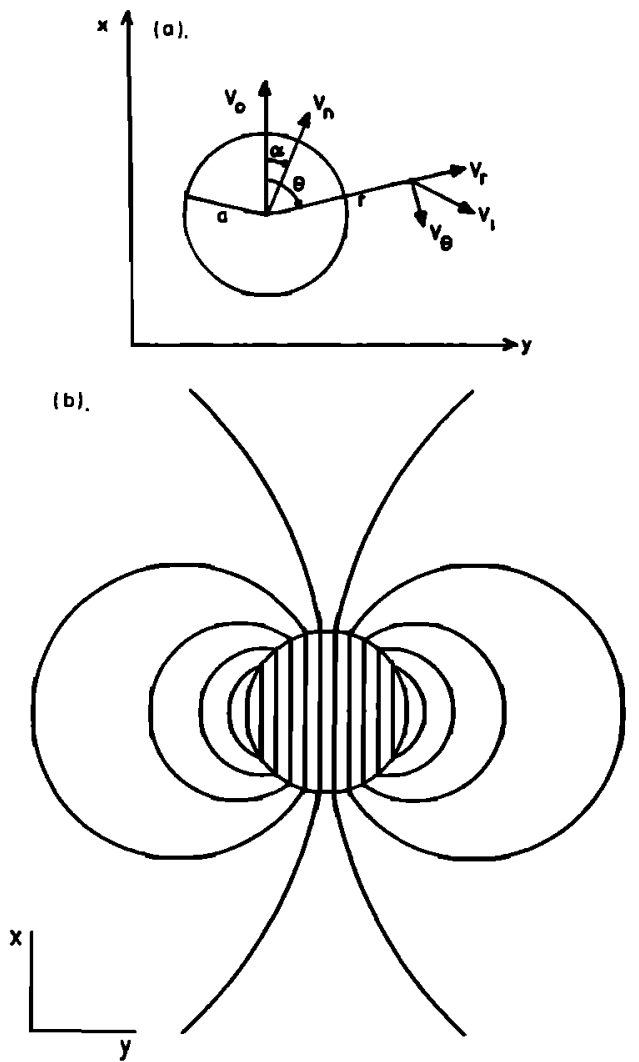

Fig. 1. (a) Geometry of flux tube velocity, $\bar{v}_{0}$, neutral wind, $\bar{v}_{n}$ and plasma flow components $v_{r}$, $v_{\theta}$. (b) Twin-vortex flow pattern in a reference frame fixed with respect to the Earth.

frame, because the pattern moves with the FTE in the $\mathrm{x}$ direction.

From (1), (3) and (4) we derive an equation for $D^{\prime}$ for all points within the open flux tube in the ionosphere:

$$
D^{\prime}=\left(v_{0}^{2}+v_{n}^{2}-2 v_{0} v_{n} \cos \alpha\right)_{(r<a)}^{1 / 2} / v_{t h}
$$

and from (1), (2) and (4), D' outside the open flux tube is

$$
D^{\prime}=\left[\frac{v_{0}{ }^{2} a^{4}}{r^{4}}+v_{n}^{2}-2 v_{0} v_{n} \frac{a^{2}}{r^{2}} \cos (2 \theta-\alpha)\right]^{1 / 2} / v_{t h}
$$

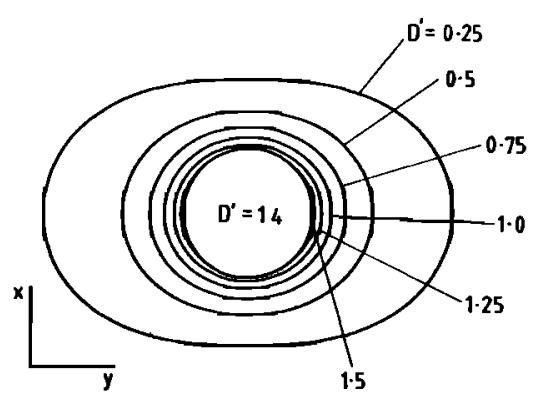

Fig. 2. Contours of $D^{\prime}$ for $v_{0}=1.5 \mathrm{~km} \mathrm{~s}^{-1}, v_{t h}$ $=1 \mathrm{~km} \mathrm{~s}^{-1}, V_{\mathrm{n}}=100 \mathrm{~m} \mathrm{~s}^{-1}$ and $\alpha=0$; within the open flux tube $D^{\prime}$ equals 1.4 .

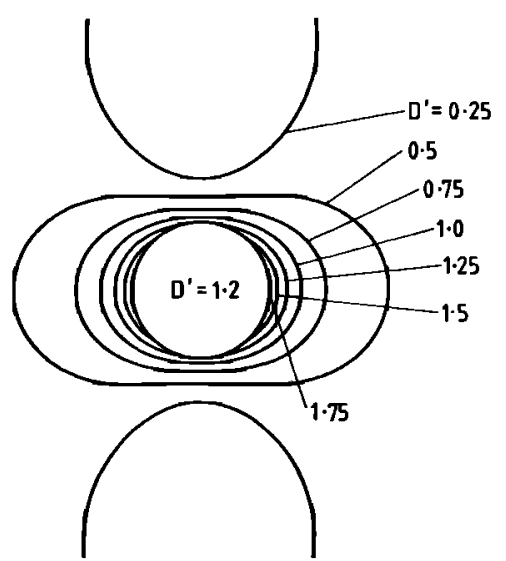

Fig. 3. Contours of $D^{\prime}$ for $v_{0}=1.5 \mathrm{~km} \mathrm{~s}^{-1}, v_{t h}$ $=1 \mathrm{~km} \mathrm{~s}^{-1}, v_{\mathrm{n}}=300 \mathrm{~m} \mathrm{~s}^{-1}$ and $\alpha=0$; within the open flux tube $D^{\prime}$ equals 1.2 .

Equation (6) gives the $D^{\prime}$ values induced by the FTE motion, they will be superposed on a much smaller $D^{\prime}$ due to the background ion flow, which is not evaluated here.

Equations (5) and (6) show that a change in the direction of the neutral wind (the angle $\alpha$ ) alters $D^{\prime}$ within the open tube region, but merely rotates the pattern of $D^{\prime}$ (by half the angle of the rotation of the wind direction) outside the FTE tube. Hence here we will, for now, only consider $\alpha=0$, i.e., wind and FTE motion in the same direction.

Figures 2, 3 and 4 show contours of $D^{\prime}$ predicted from equations (5) and (6) for $v_{n}=100,300$ and $500 \mathrm{~m} \mathrm{~s}^{-1}$ respectively with $\alpha=0, v_{0}=1.5 \mathrm{~km}$ $s^{-1}$ (roughly as observed for a twin vortex by Todd et al. [1986]), a=125 km (consistent with FTE dimension of $\sim 1 R_{E}$ at the magnetopause, Saunders et al. [1984]; Rijnbeek et al. [1987]) and $v_{t h}=1 \mathrm{~km} \mathrm{~s} \mathrm{~s}^{-1}$. The range of $v_{n}$ covers the range predicted for the throat/dayside polar cap region by Lockwood and Fuller-Rowell [1987a, b]. In all figures, contours of $D^{\prime}$ are 0.25 apart. Figure 4 shows that, for $V_{n}=500 \mathrm{~m} \mathrm{~s}^{-1}$, $D^{\prime}$ is 1.0 within the open flux tube. Outside the FTE tube $D$ ' falls of $f$ in the $x$ direction, and falls to zero where ion and neutral winds are equal and in

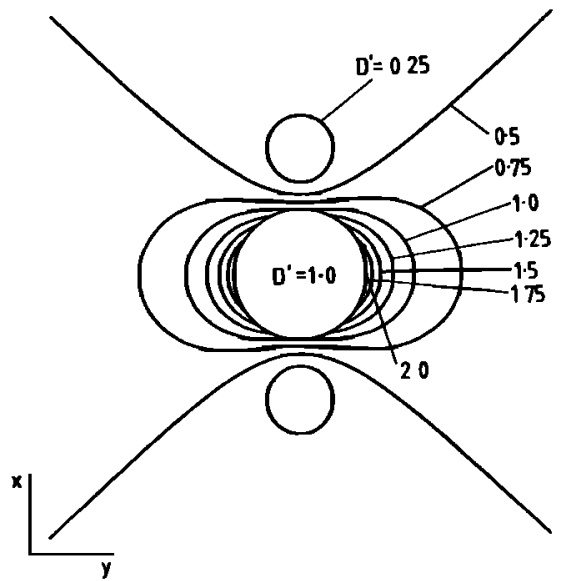

Fig. 4. Contours of $D^{\prime}$ for $v_{0}=1.5 \mathrm{~km} \mathrm{~s}^{-1}, v_{t h}$ $=1 \mathrm{~km} \mathrm{~s} \mathrm{~s}^{-1}, v_{n}=500 \mathrm{~m} \mathrm{~s}^{-1}$ and $\alpha=0$; with the open flux tube $D^{\prime}$ equals 1.0 . 
the same direction. However, on the flanks of the open flux tube the ion flow is in the opposite direction to the wind and $D^{\prime}$ exceeds 1.75 (and has a maximum of 2.0 at the points $(r, \theta)=(a, \pi / 2)$, and $(a, 3 \pi / 2))$. Hence in this case, although the plasma is near the threshold of nonthermal effects within the open tube, it is highly nonthermal just outside it and on the flanks. Hence we predict 2 regions of intense ion-neutral frictional heating and nonthermal ion velocity distribution functions just to either side of the FTE tube. For $v_{n}=300 \mathrm{~m} \mathrm{~s}^{-1}$ (Figure 3) we see similar-shaped regions of highest $D^{\prime}$ on the FTE flanks, but with lower peak values of $D^{\prime}=1.8$ and higher values within the FTE $\left(D^{\prime}=1.2\right)$, the contrast is not so great. For $v_{n}=100 \mathrm{~m} \mathrm{~s}^{-1}$ there is yet less contrast, with peak values of 1.6 outside, compared to 1.4 inside and, apart from a very narrow region on the flanks, $D^{\prime}$ is smaller everywhere outside the FTE flux tube and contours of $D^{\prime}$ are only slightly oblate for this low $v_{n}$. Hence unless the neutral wind is very low $(<1 / 10$ of the FTE velocity in the ionosphere), there will be two sizable regions of nonthermal, high ion temperature plasma to each side of an FTE flux tube (but importantly on closed field lines). Note that these can be rotated by changing the direction of the neutral wind. From the predictions of the UCL model, the directions of $\bar{v}_{n}$ in the dayside auroral oval probably limit this rotation to something less than about 30 degrees.

A similar rotation is caused if the flow is superposed on background ion flow which is not in the same direction as the FTE motion. However, as such flows are also driven by solar wind motion/ magnetic tension via reconnection to the IMF (albeit via the magnetotail, if they were connected to the IMF some time earlier), they are unlikely to be in a greatly different direction to that of the FTE motion. Southwood [1987] has pointed out that, for strong steady background convection, the FTE could form an extrusion to the polar cap which moved toward the tail round the cap boundary. In this case, one of the two vortices of the perturbation pattern would be superposed on tailward background convection inside the polar cap, the other would be superposed on the sunward, auroral return flow on closed field lines. Hence within the cap the background convection would subtract from the flow caused by the FTE, but outside the cap it would add to it. Hence a small region of even higher $D^{\prime}$ would propagate round with the bulge, just on the outside of the polar cap boundary.

\section{Transport of Upwelling Plasma within and in the wake of an FTE}

As suggested by Lockwood and Fuller-Rowell [1987a, b] and quantified by Suvanto et al. (1987), plasma subjected to high $D^{\prime}$ values will be subject to upforce due to the "hydrodynamic mirror force" and also experience transient expansion due to Joule and frictional heating, as modelled by Gombosi and Killeen [1987]. The transit times of these plasma to greater altitudes in the magnetosphere will be large, even if they subsequently undergo ion acceleration. For example, protons accelerated to $100 \mathrm{eV}$ at ionos-

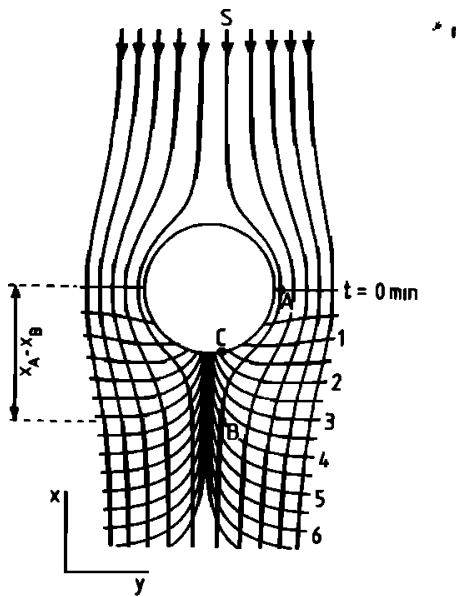

Fig. 5. Plasma streamlines (solid lines) around the open flux tube, observed in a frame moving with the open flux tube. The isochrons show the position of plasma at $30-\mathrm{s}$ intervals for $\mathrm{v}_{0}=1.5$ $\mathrm{km} \mathrm{s}^{-1}$ and $\mathrm{a}=125 \mathrm{~km}$.

pheric altitudes will still take of order $10 \mathrm{~min}$ to reach the dayside magnetopause.

Any plasma which leaves the ionosphere within the open flux tube will remain on the tube, and will hence move with the FTE, irrespective of energy and hence time of flight. Consequently, ionospheric plasma of all energies will exist between a maximum set by the peak energy of acceleration, and a minimum for which the time of flight equals the lifetime for which the FTE has been generating ion upflows. This would, for ion heating and field-aligned current effects, be the time for which the ionospheric footprint has been in motion.

Figure 5 shows the flow streamlines of plasma on closed flux tubes, in a frame moving with the FTE. The isochrons show the location of plasma at 30 second intervals. Note that the twinvortex flow model can be used to describe the gross flow features, both at the magnetopause [Farrugia et al., 1987] and also in the ionosphere [Southwood, 1985, 1987; Todd et al., 1986]. Hence Figure 5 can be applied at all heights with suitable scaling of distances and velocities. On the trailing edge of the open flux tube is a point on the boundary which moves with the FTE. Closed flux tubes which are on the boundary, $(r=a)$ of the event collect at this point and move with the FTE. Those for $r>a$ tend to bunch in the wake of the FTE. Consider one streamline, labelled $S$, on which plasma is ejected from the ionosphere at time $t=0$ at the point $A$, with energy such that its time of flight to a satellite is, for example, 4 minutes. The satellite would then intersect the ejected plasma at time $t=4$ min on a field line which projects down to the ionosphere at point B. The centre of the FTE open tube and the ionospheric source would have passed the satellite at $B, \Delta t_{A}$ minutes earlier where $\Delta t_{A}=\left(x_{A}-x_{B}\right) / v_{O}$ (see Figure 5 ), this is because the satellite moves with velocity $v_{O}$ in the frame of Figure 5. For $v_{O}=1.5 \mathrm{~km} \mathrm{~s}^{-1}$ and $a=125 \mathrm{~km}$ (as used in Figure 5), $\Delta t_{A}=2.8 \mathrm{~min}$ so the satellite would have seen the centre of the FTE at a time $t=1.2$ min. Likewise, the satellite 


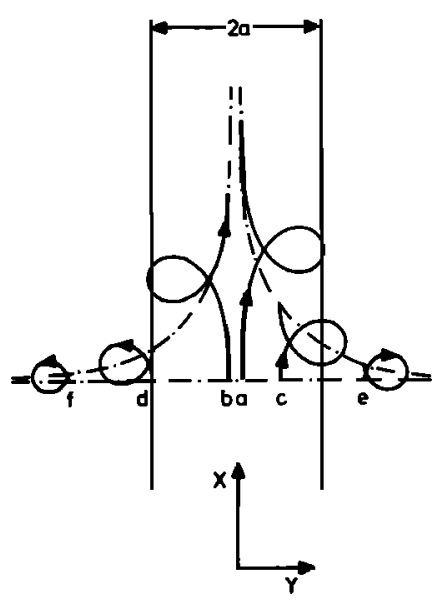

Fig. 6. Loci of flux tubes in a frame through which the FTE is moving with velocity $v_{0}$, for the same conditions as Figure 5. Loci are plotted for times $-\infty$ to $+\infty$ for initial locations $(t=-\infty)$ with $y$ of (a) $0.05 a$, (b) $-0.1 a$, (c) $0.5 a$, (d) $1.0 a$, (e) $1.5 a$, and (f) $-2.0 a$, where the FTE track (between the two vertical lines) is $2 a$ wide and centered on $y=0$. At time $t=0$ for each locus the flux tube is alongside the FTE and its displacement in the $y$ direction is a maximum. The dashed lines show the initial $(t=-\infty)$ and final $(t=\infty)$ locations of the plasma: the separation between these 2 lines (the "displacement") increases with decreasing $|y|$ and is infinite for $\mathrm{y}=\mathbf{0}$.

would see the edge of the open flux tube (at the point $C)$ at a time $t=4-\left(x_{C}-x_{B}\right) / v_{O}=2.5 \mathrm{~min}$. Hence the ions leave $A$ at $t=0$, and are seen by the satellite, at $B, 4$ min later, but the satellite saw the center of the FTE at $t=1.2 \mathrm{~min}$ and left the newly connected flux tube at $t=2.5 \mathrm{~min}$. Therefore in general, the upflow is seen a period after the FTE has passed which is less than the time of flight of the ions. The difference between these two times depends on how close to the FTE boundary the source is: for $r+\infty$ the delay equals the time of flight, for $r=a$ the delay goes to zero. The high $D^{\prime}$ regions (and field-aligned currents) associated with the FTE are near $\theta=\pi / 2$ and $3 \pi / 2$ and $r=a$, and hence we would expect to see any upflowing ions in the wake of an FTE, and after a delay which is considerably smaller than their time of flight.

In order to understand this difference between the time of flight of the ions and the delay between the observations of the FTE and the ions at the satellite, it is instructive to consider the motion of the flux tubes surrounding the FTE, in a frame which is fixed with respect to the Earth. The loci of some example flux tubes are shown in Figure 6 in this frame, for times, $t$, between plus and minus infinity where $t=0$ corresponds to the FTE center being alongside the flux tube in question (i.e., at the same $x$ ), the same definition as used in Figure 5. Note that peak $D^{\prime}$ occurs at $t=0$ (unless the neutral wind is not in the $x$ direction ie $\alpha \neq 0$ ) which is defined differently for each locus. The two straight lines in Figure 6 are $2 a$ apart and delineate the track of the FTE, and note that when $D^{\prime}$ is a maximum, each tube has moved out of the way of the FTE and is outside this track. Any plasma which encounters the exact center of the FTE $(y=0 ;(r, \theta)=(a, 0)$; see Figure $1 a)$ is moved of $f$ to infinity on the leading edge of the FTE, and similarly plasma at $(a, \pi)$ is dragged from infinity in the wake of the FTE. For original locations close to the center of the FTE track (for example $y=0.05 a$ of locus (a) in Figure 6), the nett displacement (from time $t=-\infty$ to $t=\infty$ ) is not infinite (as it is for $y=0$ ), but neither is it zero. This displacement decreases rapidly with the initial $|y|$ value. The mathematical expressions for the loci shown in Figure 6 were first evaluated by Darwin [1953] (see also MilneThompson [1955]). Note that an FTE moving round the equational magnetopause imparts antisunward momentum to closed field lines in and near to its track; hence enough such FTEs could represent a "viscous interaction" contribution to cross-cap potential. We consider that ionospheric upflows will be induced at $t=0$ and at $|y|$ close to a (i.e., on flux tubes for which $|y|$ is very close to zero before and after the passage of the FTE), as such flux tubes will experience peak ion heating and also be subject to the effects of the field-aligned currents which are required to transfer the FTE momentum from the magnetopause to the ionosphere. Note that these are the very flux tubes which are most displaced in the $x$ direction by the passage of the FTE and after launch at $t \simeq 0$, any ionospheric ions are dragged after the FTE and will be detected a certain time after the FTE; the delay being less than the time of flight, provided $\Delta y$ for the satellite (and $\theta_{1}$; see Figure 11) is very small.

Figure 7 shows how areas of ion-neutral frictional heating on closed field lines evolve, in the frame of the FTE, with height as ions move upward. The columns $(1=0,1,2,3,4)$ correspond to the heights for which the time of flight of the ions is $2.51 \mathrm{~min}$. The spatial scales have been varied so as to keep the circular open flux tube the same size. The contours shown are for flux tubes which were subject to $D^{\prime}$ values of 1.0 , 1.25 and 1.5 in the ionosphere $(1=0)$ (note the patterns for rows $A$ and $B$ and $1=0$ ) have already been discussed in Figures 2 and 4, respectively). For all cases, $v_{0}$ in the ionosphere is $1.5 \mathrm{~km} \mathrm{~s}^{-1}$ where $a=125 \mathrm{~km}$. Row $A$ is for $v_{n}=100 \mathrm{~m} \mathrm{~s}^{-1}$ with $\alpha=0$; row $B$ is for $\mathrm{V}_{n}=500 \mathrm{~m} \mathrm{~s}^{-1}$ with $\alpha=0$ and row $C$ is for $v_{n}=500 \mathrm{~m} \mathrm{~s}^{-1}$ and $\alpha=\pi / 2$. In all cases we see a tendency for the regions where heated plasma can be observed to move toward the center of the FTE track, i.e., the plasma migrates to this center as it rises ( 1 increases). Plot A4 of Figure 7 also shows two satellite paths (for satellite and magnetopause motions which are negligible compared to that of the FTE), which cut close to (1) the center and (2) the edge of the FTE open flux tube. The former will encounter highly heated plasma $\left(D_{1}{ }^{\prime}>1.5\right.$; the subscript 1 denotes that the heating took place 2.51 min earlier). Conversely, the latter never intercepts plasma which has been significantly heated $\left(D_{1}^{\prime}<1.0\right)$. Case $B$ demonstrates that increasing the neutral wind (in the direction of the FTE) gives larger values of $\mathrm{D}_{1}$ ' on the edges of the FTE track, but they are always much smaller than those near its center. Case $C$ shows that changing the wind direction $(\alpha)$ can alter the separation between the FTE and the regions of 
A

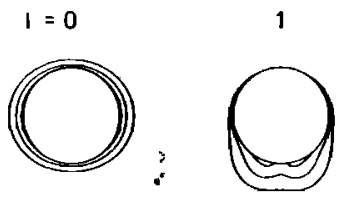

$\mathrm{B}$
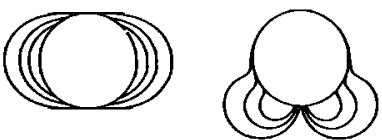

C

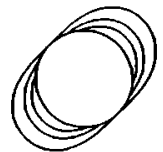

2
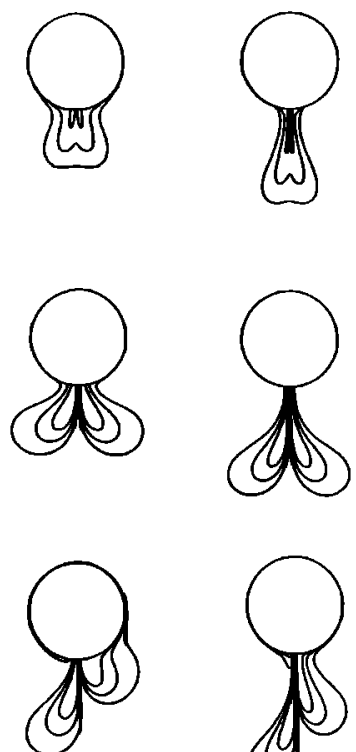<smiles>O=C1CCCCCCCCC12CCCC2</smiles><smiles>CC1C2CC1(C)CCC2(C)C1CCCC1</smiles>
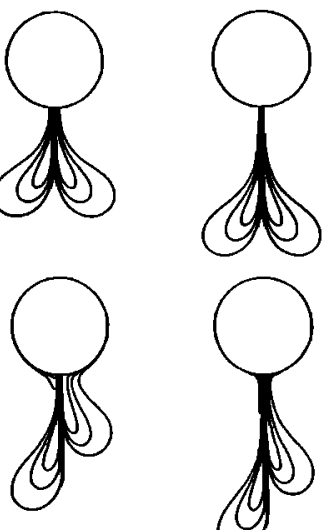

Fig. 7. Contours of $\mathrm{D}_{1}$ ' on field lines outside the FTE, which is shown in cross section by the circular region. $D_{1}^{\prime}$ is the value of $D^{\prime}$ experienced by the ionospheric foot of that flux tube 2.51 minutes earlier, which is the time of flight of the ions to the height considered. The columns correspond to 1 values of $0(1) 4$ and the rows to different neutral winds: case $A$ is for $V_{n}=100 \mathrm{~m} \mathrm{~s}^{-1}$ and $\alpha=0$; case $B$ is for $V_{n}=500 \mathrm{~m} \mathrm{~s}^{-}$ $I$ and $\alpha=0$; case $C$ is for $v_{n}=500 \mathrm{~m} \mathrm{~s}^{-1}$ and $\alpha=\pi / 2$. In the ionosphere $(1=0), v_{0}$ is 1.5 $\mathrm{km} \mathrm{s} \mathrm{s}^{-1}$ and $a=125 \mathrm{~km}$.

enhanced $D_{1}^{\prime}$, by skewing the pattern for $1=0$. Hence $\alpha$ can also influence the lag between the satellite observing the FTE and the heated ions. For example, for an FTE moving westward around the morning sector, with winds blowing northward into the polar cap, the lag is reduced for plasma on the old open flux tubes inside the cap and increased for that on closed field lines. Conversely, for an FTE in the afternoon sector the lag will be reduced on closed field lines.

Last, in Figure 8 we present some predictions of the $D_{1}$ ' values and $B_{n}$ (boundary normal magnetic field, only for outside the FTE) seen by a satellite, over which the FTE moves. The time scales are given as a fraction of the period $\Delta \mathrm{T}$ during which the satellite is inside the FTE open flux tube. Figure $8 \mathrm{a}$ is for a path which cuts the FTE near its edge $(\Delta y / a=0.9)$ with neutral wind $V_{n}=100 \mathrm{~m} \mathrm{~s}^{-1}$ and $\alpha=0$. In this case, field line draping is seen for roughly $4 \Delta T$ after exiting the FTE tube and $D_{1}^{\prime}$ starts to rise as soon as the satellite has exited the draping region and peaks at $t \simeq 8 \Delta T$. In Figure $B b \Delta y / a$ is 0.1 (ie., much closer to the centre of the track) and the angle $\alpha$ is here $\pi / 2$. The same behavior is now observed but the draping stops and the heated ion region begins near $t=\Delta T$ and $D_{1}$ ' peaks near $t=2.5 \Delta t$. In these examples $v_{o}=1.5 \mathrm{~km} \mathrm{~s} \mathrm{~s}^{-1}$ and $a=125 \mathrm{~km}$ as before, hence $\Delta T=167 \mathrm{~s}$ (independent of altitude), yet the value 1 is 5 (i.e., time of flight is $12.5 \mathrm{~min})$. Hence Figure $8 \mathrm{~b}$ shows, for example, that if $(\Delta y / a)=0.1$, we can observe ions whose time of flight is $12.5 \mathrm{~min}$, after a lag of only $\sim 3 \mathrm{~min}$ following the FTE. For yet smaller $\Delta y / a$, the lag is even shorter and high $D_{1}$ ' values are observed, contiguous with those inside the FTE open flux tube region.
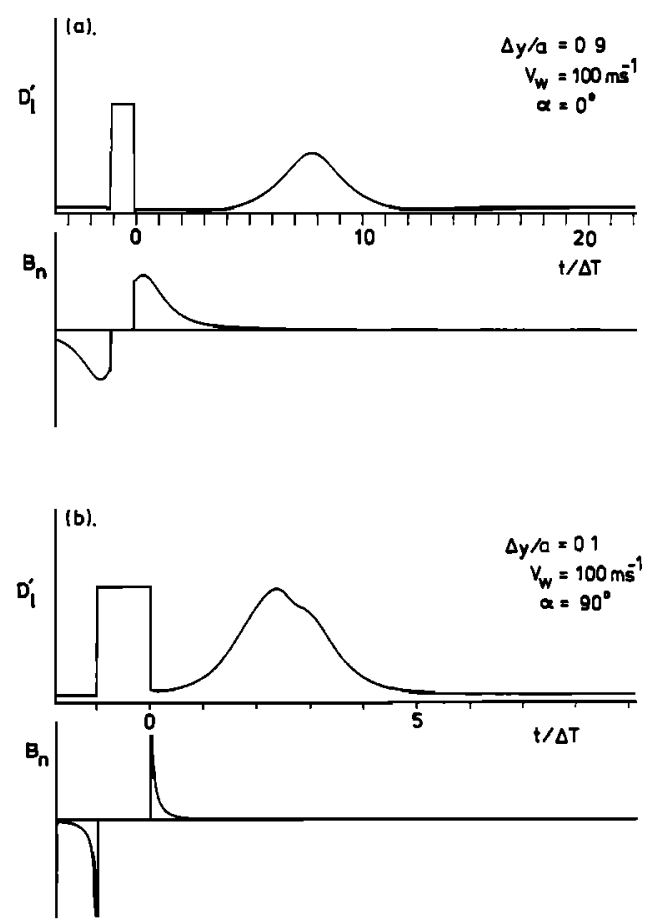

Fig. 8. Variations $D_{1}^{\prime}$ (for $1=5$, ie. time-offlight $=12.5$ minutes) and $B_{n}$ (outside the FTE connected flux tube only) observed by a satellite which intersects the FTE with (a) $\Delta y=0.9 a$, and (b) $\Delta y=0.1 \mathrm{a}$ (see Figure 11). The time ( $t$ ) axis is scaled in units of $\Delta t$, the transit time of the satellite across the FTE open flux tube. For $8 \mathrm{a}$ $v_{n}=100 \mathrm{~m} \mathrm{~s}^{-1}$ with $\alpha=0^{\circ}$, for $8 b v_{n}=100 \mathrm{~m} \mathrm{~s}^{-1}$ with $\alpha=\pi / 2$. 
4. AMPTE-UKS Observations of Upflows in the Wake of an FTE

Figure 9 shows magnetic field data from the AMPTE-UKS spacecraft on day 302 (October 28), 1984 between 1040 and 1100 UT, as observed by the UKS-magnetometer [Southwood et al, 1985]. During this period, the spacecraft observed four flux transfer events (marked A, B, C and D). The first two of these are marked by only small signatures in the boundary normal $\left(B_{n}\right)$ component, and slight rises in the dawnward, $B_{m}$, component. Events $C$ and $D$ show larger signatures. Further information on event $D$ is given in Figure 10, which is for 1040-1100 UT. For this period the spacecraft position in GSE coordinates is $X_{G S E}=$ $6.2 \mathrm{R}_{\mathrm{E}}, \mathrm{Y}_{\mathrm{GSE}}=-7.0 \mathrm{R}_{\mathrm{E}}$ and $\mathrm{z}_{\mathrm{GSE}}=0.3$, ie. close to the dayside, dawn sector magnetopause near the equatorial plane. The top panel shows the component of the $B_{n}$ magnetic field and reveals the clear FTE signature centered on 1046 UT.

Rijnbeek et al. [1987] and Farrugia et al. [1988] have also studied this event. The second panel shows the total density of plasma observed by the UKS three-dimensional ion experiment [Coates et al., 1985], integrated over a solid angle of $4 \pi$ and the entire energy range of the instrument of $\sim 10 \mathrm{eV} / \mathrm{q}$ to $20 \mathrm{keV} / \mathrm{q}$. The center of the FTE open flux tube contains magnetosheath plasma which is much denser (and of lower energy) than the magnetospheric plamsa outside the FTE [Farrugia et al., 1988]. The enhanced densities in panel 2 define the open flux tube, but note the magnetic signature extends beyond this region due to the draping of field lines around the FTE [Farrugia et al., 1987]. The third panel shows the density, $N_{f}$, observed at energies below $1 \mathrm{keV}$ for the detector bin containing the direction anti- parallel to $B, i . e .$, the density of plasma flowing up, away from the ionosphere of the nearer (northern) hemisphere. Within the open flux tube, upflowing plasma of density $\sim 0.5 \mathrm{~cm}^{-3}$ is observed. Ions are detected with energies down to the detector threshold of $10 \mathrm{ev}$, and hence there is almost certainly an upflowing ionospheric component. The bulk of the ions, however, are between $100 \mathrm{eV}$ and $1 \mathrm{keV}$, and also contains magnetosheath particles which have entered and mirrored below UKS.

No ions (above the background level) were observed before UKS entered the open tube, but immediately upon leaving it (at 1047) upflows observed with density peaking at $\sim 1 \mathrm{~cm}^{-3}$ at 1048: 30. These ions have energies of $\sim 100 \mathrm{eV}$ (compared with the $>1 \mathrm{keV}$ plasma of the surrounding magnetosphere) and form a fieldaligned upward-flowing beam, and hence appear to be ionospheric in origin. The ion detector does not have mass resolution, but assuming the ions to be protons gives a field-aligned velocity of $\sim 140 \mathrm{~km} \mathrm{~s}^{-1}$, and a time of flight of $13 \mathrm{~min}$ if they were energized near the ionosphere.

Lockwood et al. [1985a, b] have shown the dayside "throat" of the ionosphere to be a persistent source of $0^{+}$ions. If the ions detected here were $0^{+}$, the time of flight would be $52 \mathrm{~min}$. In this case they could not be associated with the observed FTEs, since they are not expected to remain on the dayside magnetopause for such a long period.

In computing the densities, $n_{f}$, in the angular bin of the detector containing the upward fieldaligned direction, it has been assumed that the angular distribution of ions fills the bin and an average over this solid angle can be used. For adiabatic, scatter-free motion of ions acceler-

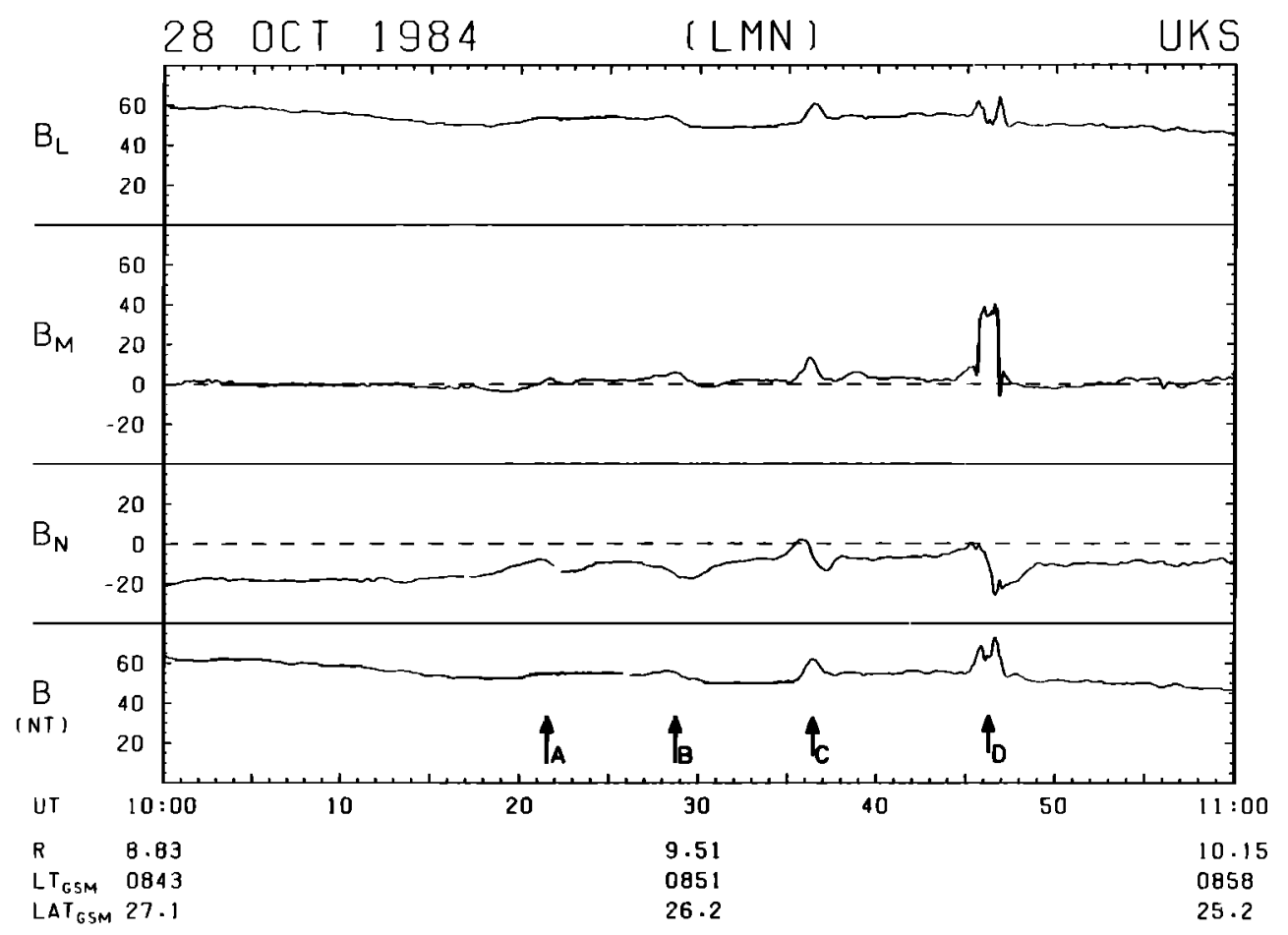

Fig. 9. Magnetic field components in boundary normal coordinates observed by AMPTEUKS between 10:00 and 11:00 UT on October 28, 1984. Flux transfer events are marked $A, B, C$ and $D$. 


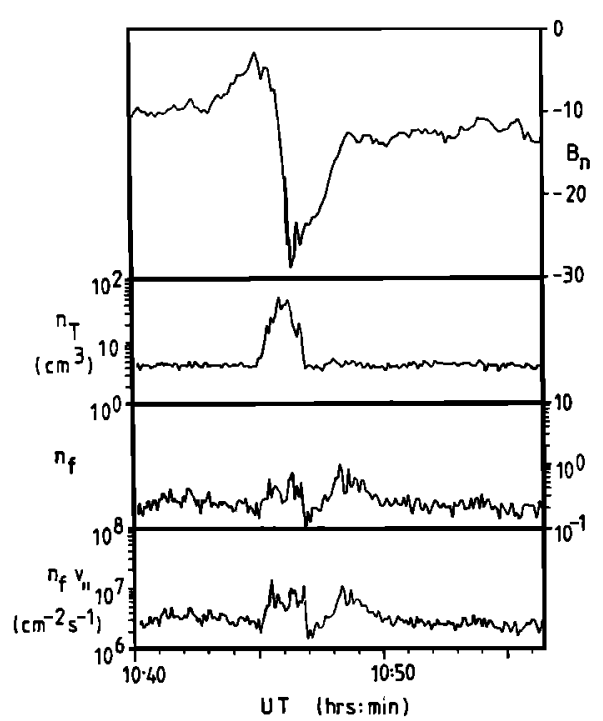

Fig. 10. AMPTE UKS observations on day 302, 1984. (a) $B_{n}$, magnetopause boundary normal magnetic field component, (showing event $D$ of Figure 9), (b) total plasma density, $\mathbf{N}_{T}$, (c) ion density for ions flowing antiparallel to $B, N_{f}$, and (d) the flux of ions antiparallel to $B, N_{f}$ $\mathrm{V}_{\|} \cdot$

ated in the ionosphere, the beam at the equation magnetosphere would have considerably smaller angular width than the detector bin and this would cause $n_{f}$ (and the flux $n_{f} \cdot v_{\|}$) to be underestimated, possibly by several orders of magnitude. However, a small tail to the angular distribution of these ions is observed in adjacent angular bins and hence we conclude that this is not occuring. Note that this calls for some scattering or heating processes at low magnetospheric latitudes which act to broaden the distribution.

Hence this example shows ion upflows, which are probably ionospheric in origin, observed in the vicinity of some FTE, but on closed field line outside the events. The upflows are only observed after the FTEs have passed, but with a delay of $4.5 \mathrm{~min}$ from the center of event $D$ and $12.5 \mathrm{~min}$ from that of event $C$. The time of flight of the ions (assumed to be $\mathrm{H}^{+}$) from the ionosphere would be roughly $13 \mathrm{~min}$. A source and energization altitude above the ionosphere would reduce the time of flight estimate. Note, however, that an ionsopheric source with energization at a greater altitude would increase the time of flight, as ions would travel further at lower energies. From the considerations in the previous section, the ionospheric ions can only be found within the FTE wake if the footprint has been in motion for a period greater than the time of flight. For the location of the observed FTE, well away from noon, this seems entirely possible in this case, but we must first consider this possibility quantitatively. Subsequently, in section 4.2, we will discuss which FTE could have generated the ion upflows.

\section{1 Time of Flight and the Lifetime of the FTEs}

In order to estimate how long each of these FTEs had been on the magnetopause, let us assume the reconnection event took place at a subsolar point $\mathrm{X}_{\mathrm{GSE}}=9.35 \mathrm{R}_{\mathrm{E}}, \mathrm{Y}_{\mathrm{GSE}}=0, \mathrm{Z}_{\mathrm{GSE}}=0$ (which requires the dayside magnetosphere to be circular in the equatorial plane), in which case the FTE has moved through $8 R_{E}$ from the subsolar point to the point of observation by UKS. The velocity of the event $D$ at UKS in the boundary, L-M plane is estimated to be $\sim 130 \mathrm{~km} \mathrm{~s}-1$ at $51^{\circ}$ to the $L$ axis. [Smith et al., 1987]. Hence the component of the velocity in L direction (toward dawn) is $100 \mathrm{~km}$ $\mathrm{s}^{-1}$ : if this value had remained constant between the reconnection event and the observation, the FTE would have been moving for $\sim 8.5 \mathrm{~min}$. This is lower than the $\sim 13 \mathrm{~min}$ time of flight of the ions. However, if we assumed that the FTE was initially at rest and suffered constant acceleration until the time of observation, then the FTE would have been moving for roughly $17 \mathrm{~min}$. We conclude that the FTE has been moving for somewhere between 8.5 and $17 \mathrm{~min}$. In addition, considering the uncertainties in the point of reconnection, the satellite-to-ionosphere distance and the possible variation in the velocity of the FTE, it does indeed seem probable that this FTE had been in existence for longer than the time of flight of the ions. We note that few ions at energies much below $100 \mathrm{eV}$ were observed, suggesting the lifetime of the FTE did not greatly exceed the time of flight of the 100 eV ions, and lower energy ions have had insufficient time to reach UKS.

There are two components of an FTE's motion on the magnetopause: magnetosheath flow and the effect of magnetic tension; The latter causes motion at roughly the Alfven speed and, because in this case the sheath flow is super-Alfvenic ( $150 \mathrm{~km} \mathrm{~s} \mathrm{~s}^{-1}$, Rijnbeek et al. [1987]), it is expected that this will control the FTE motion, and indeed the deduced speed and direction of this FTE is consistent with the magnetosheath flow observed by UKS. Hence this event is moving dawnward around the polar cap boundary and magnetopause, as suggested by Cowley [1984].

It should also be noticed that if the FTE moved purely in the $L$ direction after reconnection ( $B_{y}=0$ and dominant tension effects), there may be no movement of the ionospheric foot of the open tube, and hence no ionospheric twin vortex signature, until the field line has straightened and the FTE is no longer on the magnetopause [Southwood, 1987]. Motion in the M direction should give ionospheric motion (around the cap boundary) almost immediately.

\section{2 Which FTE Generated the Ion Upflows?}

The ions are observed more than 21 and $27 \mathrm{~min}$ after the centers of the small events $B$ and $A$, respectively. As these delays are considerably greater than the predicted time of flight of the ions and greater than the estimated range of the lifetimes of the FTEs on the dayside magnetopause, presented in section 5.1, we conclude that they cannot have generated the ion upflows. The delay for event $C$ is roughly equal to the time of flight (12.5 min) but that for event $D(2.5 \mathrm{~min})$ is much smaller. The discussion in section 3 demonstrates that the delay can be considerably smaller than the time-of-flight, provided that the satellite lies very close to the centre of the FTE track.

The separation of the track center and the satellite for event $D$ has been studied using the 


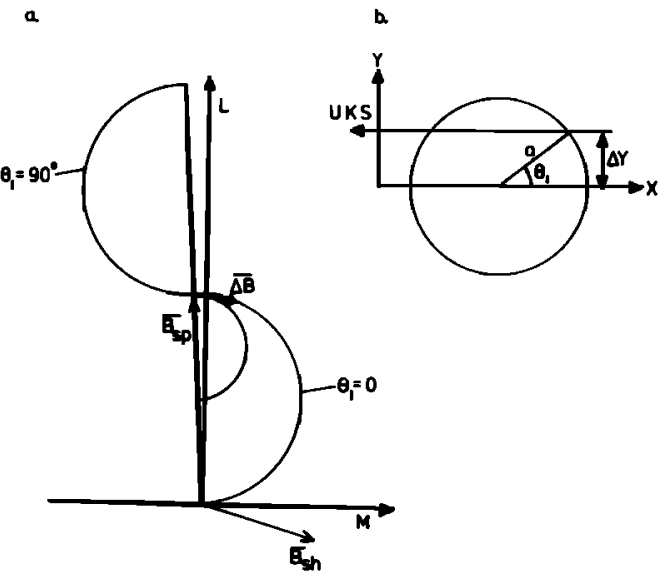

Fig. 11. (a) Magnetic fields in the notional magnetopause plane as viewed from the Earth. The ambient field configuration $\left(\mathrm{B}_{\mathbf{s p}}, \mathrm{B}_{\mathrm{sh}}\right.$ ) are drawn to scale and 3 circular arcs are shown. The vector $\overline{\Delta B}$ represents the magnetic field perturbation in the draping region and is taken from the UKS data, for event $D$ (see Figure 9). This shows that the spacecraft intersects the magnetospheric arm of the open flux tube, very close to its center $\left(\theta_{1}=0\right)$, see part $\left.b\right)$. For $0<\theta_{1}<45^{\circ}$ the arc has a smaller radius (one arc is shown for this range of $\theta_{1}$ ) while for $\theta_{1}>45^{\circ}$ the arc would move to higher, not lower, $L$ vaues. It can be seen that for this event (D), a very small $\theta_{1}$ gives the correct size and sense of the perturbation $\overline{\Delta \mathrm{B}}$.

method of Farrugia et al. [1987]. From the ion data we see an order of magnitude rise in the plasma density, $\mathrm{N}_{\mathrm{T}}$, (see Figure 10b) and an order of magnitude fall in ion temperature (not shown), hence the satellite is certainly within the open flux tube track of event $D$, and is found to be on the magnetospheric "arm" of the event. High resolution magnetometer data for this event (see Figure 1 of Farrugia et al. [1988] and Rijnbeek et al. [1987]) show that the perturbations in the draping region are predominantly in $\mathrm{B}_{\mathrm{M}}$ and $\mathrm{B}_{\mathrm{N}}$ : $\mathrm{B}_{\mathrm{M}}$ increases by about $10 \mathrm{nT}$ and $\mathrm{B}_{\mathrm{N}}$ increases (becomes less negative) by the same amount, whereas there is very little change in $B_{L}$. Using the Farrugia et al. [1987] diagnostic method, based on the same model of incompressible flow around a flux tube as employed in section 3 , we derive the construction shown in Figure 11. The ambient sheath field used is the one observed at the main magnetopause crossing around 1210 UT ( $B_{S h}=30 \mathrm{nT}$, whereas $B_{\mathrm{SP}}$ (inside the magnetopause) $\simeq 55 \mathrm{nT}$ ). Figure $11 \mathrm{~b}$ shows the orientation of the satellite track with respect to the FTE, and defines the angle $\theta_{1}$. If an observer moved along the reconnected flux tube the field perturbation, $\Delta B$, would lie along a circular locus, shown in Figure $11 \mathrm{a}$ for $\theta_{1}=0^{\circ}, \theta_{1}=\pi / 2$, and a $\theta_{1}$ valve between 0 and $\pi / 4$. The observed $\Delta B$ lies on the curve for $\theta_{1}=0$; $1 . e ., \Delta y \simeq 0$. Hence the draping $\Delta B$ shows that the spacecraft trajectory passes close to the center of the FTE open flux tube. However, the increase in $\mathrm{B}_{\mathrm{M}}$ inside the open flux tube (Figure 9) shows that it does not move precisely through the center. We estimate that the modulus of $\Delta y$ is about $0.1 \mathrm{a}$. Hence we must conclude that event $D$ could indeed be the source of the ions, from the arguments presented in section 3.
The plasma data for event $\mathrm{C}$, conversely, show no major increase of $\mathrm{N}_{\mathrm{T}}$ and no decrease in temperature. Nevertheless, a detailed study of the ion spectrogram reveals the possibility that the satellite touched the open flux tube of event $C$, but for a brief period. We conclude $\Delta y \simeq a$ for this event. From the argument presented in section 3, this should result in a delay between the FTE center and the observation of any ejected ionospheric ions which is only very slightly smaller than the time of flight.

From these arguments, it is not possible to tell if the upflows were generated by event $c$ or event $D$. However, it is important to consider the implications of the ionospheric source region for these two possibilities. If we consider the source to be event $D$, the ions would have arisen very close to the flanks of the FTE, in order that they be dragged into the very centre of the FTE wake (see Figures 5 and 7 for $1=4$ ). This region is one of peak $D^{\prime}$ (see Figures 2-4) and is also expected to be the site of the field-aligned currents. Conversely, if event $c$ is the source the ions will have arisen from much further away from the FTE track $(|y|>a)$ to be observed at $\Delta y=a$. The $1=4$ column of Figure 7 demonstrates that $D_{1}$ ' is low for satellite path $(7 b)$, for which $\Delta y=a$, and this region is also not associated with strong field-aligned currents. Motion of the magnetopause (variation of $\Delta y$ with time) cannot be invoked because in order to see ions from the very edge of the FTE track, $\Delta y$ must approach zero and ions would not be seen after a delay which was as large as the time of flight. Hence from this consideration of the most likely ionospheric source, the authors believe that the event $D$ was indeed responsible for generating the ionospheric ion upflows.

Rijnbeek et al. [1987] have pointed out that, when viewed at very high resolution, this event does not look like a flow around an undeformable object, with a well-defined, sharp boundary, but there are boundary effects in a layer $\sim 1 R_{E}$ wide at the magnetopause. The authors, however, conclude that by 1047:10 UT, UKS is out of this boundary layer and on closed field lines. Hence the ion upflows are not within this event boundary layer (see figure 5) but are on closed field lines entirely outslde this FTE. This boundary will be discussed again in relation to some EISCAT observations, in section 5 .

\section{3 Consequences for the Ionosphere and the Low- Latitude Boundary Layers}

The bottom panel in Figure 6 shows the flux of ions observed by UKS to be flowing antiparallel to the field. Both within the open flux tube and in the upflowing ion event following the FTE, the flux is roughly $10^{7} \mathrm{~cm}^{-2} \mathrm{~s}^{-1}$. If the flows are ionospheric in origin, this calls for a flux of $10^{10} \mathrm{~cm}^{-2} \mathrm{~s}^{-1}$ out of the ionosphere. This is two orders of magnitude larger than that predicted for the "classical" polar wind, i.e., the outflow driven by typical electron and ion temperatures and density (see reviews by Lockwood [1986], Schunk [1986]). In order to explain these outflows, therefore, additional effects are required, such as those discussed earlier driven by time-dependent response to high $D^{\prime}$ values.

It is worth noting that this outflow rate could be highly significant in terms of ionos- 
pheric densities. If we take a typical dayside auroral TEC (total electron content) to be $\sim 10^{13}$ $\mathrm{cm}^{-2}$, we require only $10^{3}$ seconds to remove the entire ionopshere if the flow rate is maintained. The extent of the ionospheric region responsible for the outflow on closed field lines is not clear, but it will be of order of the FTE radius, $a$, and for $a=125 \mathrm{~km}$ and $v_{0}=1.5 \mathrm{~km} \mathrm{~s}^{-1}$ we can estimate the ionospheric outflow may persist for of order $100 \mathrm{~s}$ on each flux tube. In this case the observed flow would cause a 108 depletion of plasma density which would appear in a thin band in the center of the FTE wake. For lower values of the total content, more severe outflow depletions will be seen, if the same flow rate can be sustained. This gives rise to the possibility of FTEs being detected by a linear ionospheric depletion left in their wake. The depletion of the ionosphere on the open flux tube inside the FTE is even greater. We have deduced the source FTE to have been in motion for of order $15 \mathrm{~min}$, which is sufficient to completely deplete the ionosphere inside the FTE for an initial TEC of $10^{13} \mathrm{~cm}^{-2}$. Hence an additional possibility is to

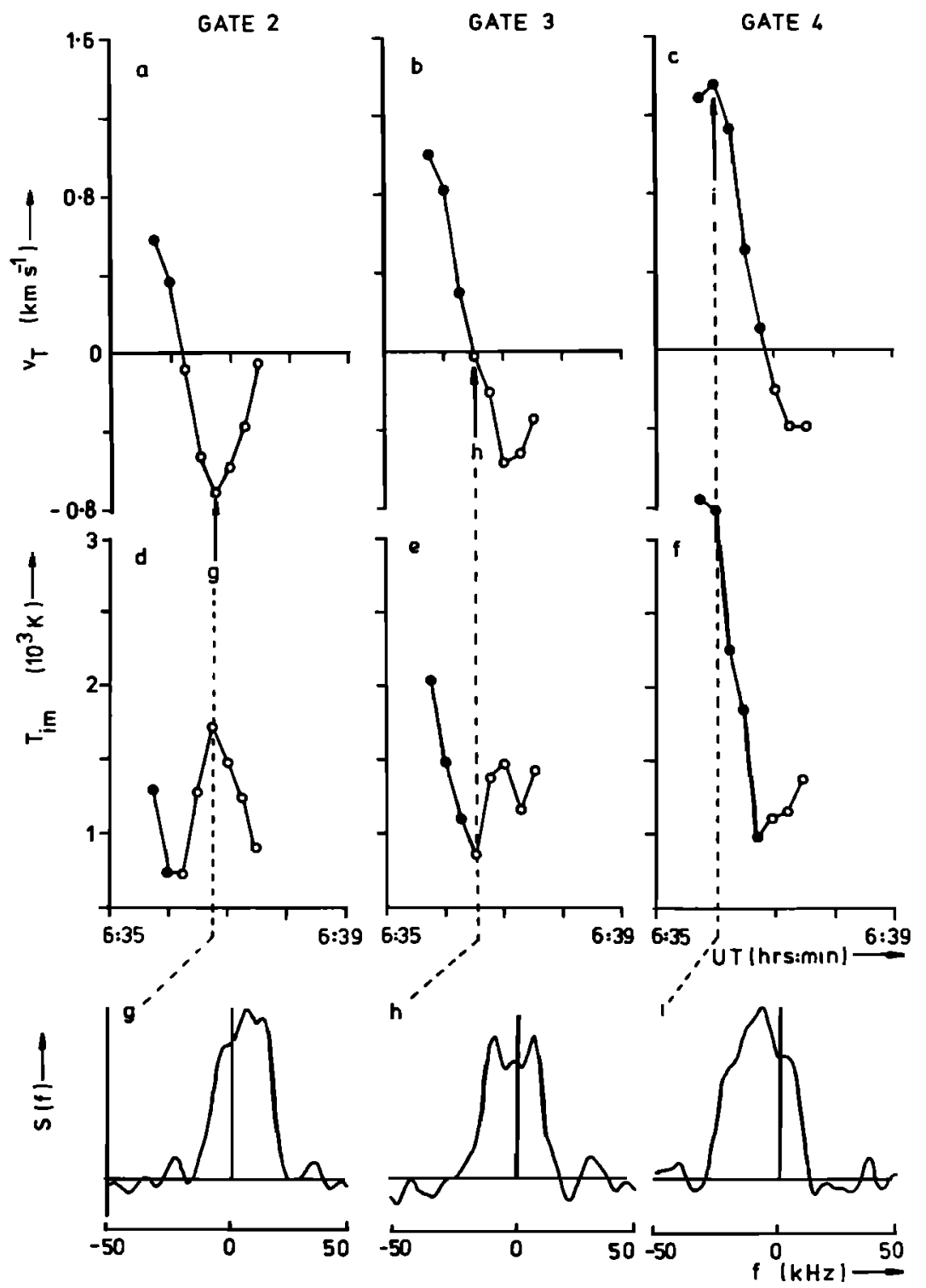

Fig. 12. Fifteen-second EISCAT observations using UK-POLAR, of a flow burst event on October 27, 1984 which are fully consistent with a twin-vortex ionospheric signature of an FTE [Todd et al., 1986]. Line-of-sight velocities, $v_{T}$, are shown for (a) gate 2 (invariant latitude, $\left.\Lambda=71.4^{\circ}\right)$; (b) gate $3\left(\Lambda=72.0^{\circ}\right)$; (c) gate $4\left(\Lambda=72.6^{\circ}\right)$ : (d), (e) and (f) show the corresponding apparent ion temperatures, $T_{i_{m}}$ (see text) for these gates. Solid points are for flows away from the radar (poleward), open circles are flows toward the radar (equatorward). Spectra for 3 data points are shown below. (g) For gate 2 at 0636:45 UT. (h) For gate 3 at 0636:15 UT. (i) For gate 4 at 0636:00 UT. Both peak poleward flows (i) and peak equatorward flows (g) give spectra characteristic of nonthermal plasma [Lockwood et al., 1987] but not the low-flow region at the boundary $(\mathrm{h})$. 
look for severe depletions of the dayside polar $F$ region which are of order $100 \mathrm{~km}$ in radius and are convecting poleward or around the polar cap boundary, deepening as they propagate. Note that the flux of $10^{10} \mathrm{~cm} \mathrm{~s}^{-1}$ deduced here may be an underestimate both because it does not include ions of energy above the gravitational escape energy but which had not reached UKS by the time of observation, and also because it has been assumed that the ion beam has filled the solid angle bin of the detector.

These ionospheric upflows also give a mechanism whereby the low-latitude boundary layers on closed field lines can be populated with ionospheric ions. The term "boundary layers" is used loosely here to mean both the low-latitude boundary layer (LLBL) and/or the halo [Sckopke et al., 1981; Challoner et al., 1987]. Estimating how many such ions are injected by each FTE in total also depends upon how wide the ionospheric upflow region is on the edges of the FTE track. Consistent with our belief that event $D$ is the source in the example discussed here $(\Delta y<0.1 \mathrm{a})$, we speculate that a value of $10 \mathrm{~km}$ may be appropriate, giving a total of $10^{26}$ ionospheric ions per FTE $\left(10^{10} \mathrm{~cm}^{-2} \mathrm{~s}^{-1}\right.$ over an area of $10 \mathrm{~km}$ by $100 \mathrm{~km}$ for $10^{3} \mathrm{~s}$ ) if it remains on the magnetopause for $15 \mathrm{~min}$. The significance of this figure depends on the average frequency of FTEs. In addition, the FTE studied here may not be "typical" in that it generates an ionospheric outflow flux of $10^{10}$ $\mathrm{cm}^{2} \mathrm{~s}^{-1}$ and remains on the magnetopause for $\sim 15$ min. Ions deposited by this mechanism would form a thin shell at the centre of the FTE track, which may well move across the LLBL, through the halo and into the inner magnetosphere. Hence this mechanism could well explain some of the complex structure seen in the boundary layers. Note that it is likely that a roughly equal number of ionospheric ions $\left(10^{26}\right)$ could be deposited in the magnetosheath on the open field lines on the other side on the FTE.

\section{EISCAT Observations of Nonthermal Ionospheric Plasma in Association with an FTE Flow Signature}

Todd et al. [1986, 1987] have reported EISCAT observations using the UK Special Program UKPolar, of flow bursts which are consistent with transient twin vortex flow patterns in the $F$ region ionosphere. This is the expected ionospheric signature of FTEs [Southwood 1985, 1987]. Lockwood et al. [1987a, b] have shown that the high ion velocities in the center of such an event drive the plasma into non-Maxwellian ion velocity distributions. Figure 12 summarizes some of the features of an event on October 27, 1984, already discussed by Todd et al [1986] and Lockwood et al. [1987a]. For the three gates shown, we see a reversal from poleward to equatorward flow, interpreted by Todd et al. [1986] as the open flux tube region boundary passing poleward over the scattering volumes. The spectra in parts $12 \mathrm{~g}$ and $12 \mathrm{i}$ are indicative of non thermal plasma, with a dominant central peak and only smaller shoulders at the ion accoustic frequencies, as predicted by Raman. et al [1981] and confirmed by Hubert [1984]. These spectra are expected when $D^{\prime}$ exceeds some thres- hold between about 1 and 1.5, and hence Figure 12 is consistent with the predictions of $D^{\prime}$ in Figures 2-4 in that nonthermal effects are apparent both inside the open flux tube (in the poleward flow. region) and outside it, on closed field lines (the equatorward flow region). Furthermore, spectra characteristic of nonthermal plasma appear only when $V_{T}$ exceeds about $1 \mathrm{~km} \mathrm{~s}^{-1}$ in the poleward flow region, whereas Figure 12d shows such effects when $V_{T}$ is only $-600 \mathrm{~ms}^{-1}$. It is, of course, possible that there is a cross beam velocity component (perpendicular to $v_{T}$ ) which could cause this difference. However, Lockwood et al. [1987b] have deduced this unseen component to be small for this event, in which case we could infer a poleward neutral wind, $v_{n}$, of roughly $200 \mathrm{~m} \mathrm{~s}^{-1}$ as the cause of this asymmetry, similar to the predictions in Figure 3. Lockwood et al [1987a, b] have been able to exploit the relatively large number of data points in the poleward flow region by using regression fits to show that the plasma is indeed nonthermal as the spectra suggest. However, insufficient data points exist in strong equatorward flow regions to allow the application of the same statistics to the observations on closed field lines.

It is interesting to note some boundary effects in these EISCAT data. The velocity, $V_{T}$, is a weighted mean value when a velocity shear (boundary) is within the scattering volume. (R. B. Horne, private communication [1987]). Hence the gradual (within $\sim 75$ s for each gate) reversal in $v_{T}$ could be caused by a sharp boundary moving through the scattering volumes (which are $75 \mathrm{~km}$ long) at $v_{0} \sim 1 \mathrm{~km} \mathrm{~s}{ }^{-1}$. Hence the $V_{T}$, line-ofsight velocity, data are not inconsistent with a sharp boundary of the kind used in our model (Figure 1b). However, the apparent ion temperature, $T_{i m}$, shows a minimum at the zero crossing of $V_{\mathrm{T}}$. As discussed by Lockwood et al [1987b], this does imply a minimum in the real ion temperature, $T_{i}$, at the boundary and hence a decrease in the magnitude of the velocity at the boundary. Hence the EISCAT data do show some evidence for an event boundary layer, similar to that detected by Rijnbeek et al. [1987] for the magnetopause signature of the FTE discussed in section 4.

\section{Discussion and Conclusions}

There is one other, entirely independent, piece of evidence for FTE wake effects, of the kind described above. If the motion of closed flux tubes shown in Figure 13 is transposed into a frame which is fixed with respect to the Earth, Figure 8 is obtained. Consider a circular FTE (of radius $125 \mathrm{~km}$ ) being formed to straddle the polar cap boundary. By $t=0$ the re-connection has ceased and the boundary of open and closed flux tubes is as shown in Figure 13, with a semi circular equatorward extrusion of the polar cap. Figure 13 then shows the effect of the FTE subsequently moving at right angles to the boundary with constant velocity of $1.5 \mathrm{~km} \mathrm{~s}^{-1}$. Note that motion parallel to the boundary merely causes the extrusion to move along the boundary, as described by southwood [1987]. The boundary is shown at 1 min intervals. It can be seen that as the twin vortex pattern moves through $i t$, the 


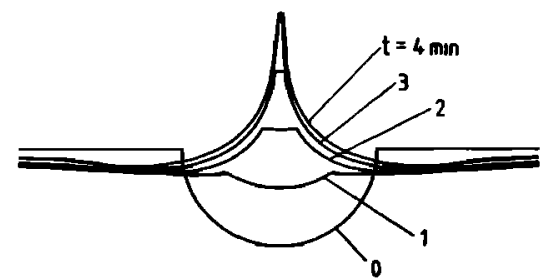

Fig. 13. Boundary between open and closed field lines at $1-m i n$ intervals for $a=125 \mathrm{~km}$ and constant $\mathrm{v}_{0}=1.5 \mathrm{~km} \mathrm{~s} \mathrm{~s}^{-1}$ at right angles to the old polar cap boundary.

boundary moves equatorward outside the event, but a sharp cusp-shaped feature forms inside the FTE track and moves poleward of the old boundary position. Events in the dayside cusp, observed by optical ground-based all-sky cameras have recently been described as the ground signature of FTEs, by virtue of their duration and size, by Sandhold et al. [1986, 1987]. These events show similar behavior to that predicted here, with an equatorward extrusion in the optical emissions being followed by a poleward retreat at the event center to a position poleward of that before the extrusion formed. The optical luminosity behavior is therefore explained by the particle precipitation remaining close to the boundary of open and closed field lines. It is on this cusp of luminosity that the ion upflow predicted here would tend to be bunched at greater altitudes by time-of-flight effects. The length of the "cusp" may well be limited by the fact that as the FTE moves poleward it is subsumed into the polar cap flow and the twin vortex flow weakens.

It is interesting to note that both FTEs and ionospheric plasma upwellings are highly regular features of the cusp. The upwelling ions [Lockwood et al. , 1985a, b, c; Moore et al., 1986] are always observed and hence the "cleft ion fountain" they form is almost certainly continuous and therefore may not be satisfactorily explained as due to a series of FTEs. However, an FTE would certainly enhance the cleft ion fountain by the processes discussed here. In the upwelling ion event discussed by Moore et al. [1986] the source region mapped to an intense sunward flow "jet", just equatorward of the polar cap boundary. The magnitude of the flow in this jet $\left(\sim 6 \mathrm{~km} \mathrm{~s}^{-1}\right)$ is much larger than expected for steady convection in the dawn auroral oval and hence it is possibly enhanced by an FTE moving around the dawn polar cap boundary. As discussed in section 2, this would give an exceptionally large $D^{\prime}$ immediately equatorward of the cap boundary, but would cause upwelling plasma to be moved onto the cap boundary in the wake of the extrusion as it moved around toward dawn or dusk (in the manner discussed in section 3). Hence upwelling plasma is produced in exactly the position required by the magnetospheric observations of $0^{+}$ions [Lockwood 1985a, b; Moore, 1986], being moved onto open field lines in the throat region and subsequently convected into the polar cap in the "cleft ion fountain". Note also that the asymetry in morning and afternoon sector auroral neutral winds would tend to give higher $D^{\prime}$ in the morning sector, consistent with the observed dawn-dusk asymmetry of upwelling ion events [Lockwood et al. 1985].
The model employed assumes a sharp boundary to the open flux tube and laminar flow around it. The same assumptions were made by Farrugia et al. [1987] who were consequently able to reproduce the observed field line draping at the magnetopause. We note that a wide boundary region and/or eddies in the FTE wake will have consequences for both $D^{\prime}$ and ion trajectory predictions. Nevertheless, the model does offer explanations of nonthermal plasma on apparently closed field lines observed by EISCAT, and of low-energy upflows in the wake of an FTE observed by AMPTE-UKS.

Figure 14 summarizes the proposed mechanism whereby an FTE drives ion outflows on closed field lines, which we have proposed in this paper. As the FTE moves around the magnetopause, it initially moves a flux tube (which is close to the center of its track) ahead of it, as shown in Figure 14a. At some time the field line will move round the side of the FTE, the ionospheric foot experiencing peak heating at this time and we expect the ionospheric ions to begin to travel along the field line (Figure $14 \mathrm{~b}$ ). The subsequent plots show the flux tube, now containing ionospheric ions, being dragged after the FTE and back into the center of the FTE's track, in its wake. By the time shown in part Figure 14c, the ions have arrived at the satellite in the equatorial boundary layer.

Last, we note there has been much interest recently in hot electrons which are found on the edges of the FTE connected flux tube [Scudder et al. 1984]. In order to explain the continued energization of these electrons, and the fact that FTEs are statistically larger at greater distances from the subsolar magnetopause,
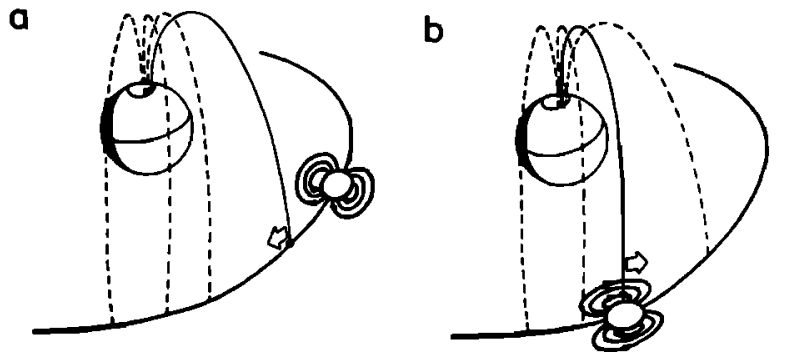

c
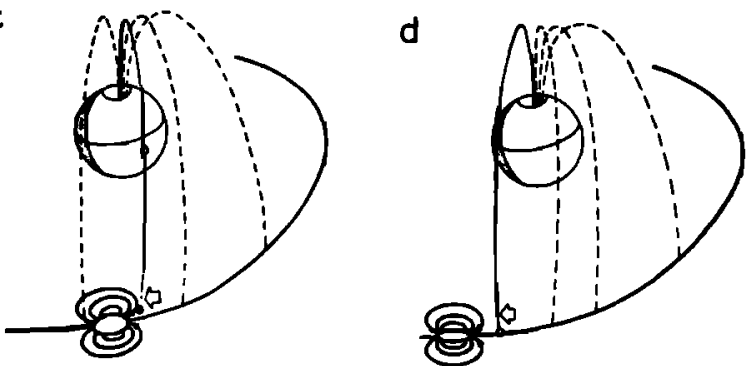

Fig. 14. Schematic of the proposed ion outflow mechanism due to the twin-vortex flow pattern of an FTE as it moves around the magnetopause (the solid curve). The solid line shows the closed field line at each time in the sequence and the dashed lines its location at the other times for comparison. The open circle shows the location of the first (highest energy) upflowing ionospheric ions. 
Sonnerup [1987] and Southwood et al. [1988] have postulated continuing reconnection mechanisms as the FTE propagates. These theories have implications for the work presented here (which makes use of the Russell and Elphic [1978] model, where reconnection only occurs for a brief period), but do not invalidate any of the principles in that a magnetically connected region will still be dragged through the ionosphere and magnetosphere, pushing closed and "old" open field lines out of its path. The southwood et al. picture would allow this region to be substantially larger (in the $y$ direction) than is assumed here and would increase the area of the region from which ionospheric upflows are driven, and hence would allow larger total ion outflows.

Acknowledgments. The authors are grateful to D. J. Southwood for the provision of AMPTE-UKS magnetometer data, and to W. C. Mier-Jedrzejowicz and B. J. I. Bromage for processing the UKS magnetometer and UK-POLAR EISCAT data, respectively. We would also like to give thanks to $R$. C. Elphic, A. D. Johnstone, D. J. Southwood, S. W. H. Cowley, D. M. Willis, K. Winser and N. Murphy for most helpful discussions of this work, and to the Director and staff of EISCAT for their help. EISCAT is supported by the British SERC, French CNRS, West German MPG, Norwegian NAVF, Swedish NFR and Finnish SA. AMPTE is a collaborative project of NASA (USA), MPG (West Germany) and SERC (UK). The work at UCLA was supported by a grant from the US National Science Foundation (ATM 85-03980).

The Editor thanks R.C. Elphic and T. E. Holzer for their assistance in evaluating this paper.

References

Barakat, A. R., R. W. Schunk, and J.-P. St.Maurice, Monte Carlo calculations of the $0+$ velocity distribution in the auroral ionosphere, J. Geophys. Res., 88, 3237-3241, 1983.

Barakat, A. R., and R. W. Schunk, Ef fect of hot electrons on the polar wind, J. Geophys. Res., 89, 9771-9783 (1984).

Chaloner, C. P.,.D. R. Lepine, D. S. Hall, D. A. Bryant, W. Studeman, B, Wilken, G. Kremser, F. Sфraas, K. Brфnstad, J. F. Fennell, B. Blake, $R$. Koga, and R. Lundin, The formation of boundary layers by the entry of low energy electrons and energetic ions into the earth's magnetosphere, Proceedings of 21st ESLAB Symposium, Bolkesjo, Norway, Eur. Space Agency Spec. Publ. ESA SP-275, 51-56, 1987.

Coates, A. J., J. A. Bowles, R. A. Gowen, B. K. Hancock, A. D. Johnstone, and S. J. Kellock, The AMPTE UKS three-dimensional ion experiment, IEEE Trans. Geosci. Remote Sens., GE-23, $287-292,1985$

Cowley, S. W. H., Evidence for the occurrence and importance of reconnection between the Earth's magnetic field and the interplanetary field, in Magnetic Reconnection in Space and Laboratory Plasmas, Geophys. Monogr. Ser., vol. 30, edited by E. W. Hones, Jr., pp.375378, AGU, Washington, D. C., 1984.

Darwin, C., Note on hydrodynamics, Proc. Cambridge Philos. Soc., 49, 342-354, 1953.

Dusenbery, P. B., and L. R. Lyons, Generation of ion-conic distribution by upgoing ionospheric electrons, J. Geophys. Res., 86, 7627-7638, 1981.

Farmer, A. D., M. Lockwood, T. J. Fuller-Rowell, K. Suvanto, and U. P. Lovhaug, Model predictions of the occurrence of non-Maxwellian plasmas, and analysis of their effects on EIscat data, J. Atmos. Terr. Phys., in press, 1988.

Farrugia, C. J., R. C. Elphic, D. J. Southwood, and S. W. H. Cowley, Field and flow perturbations outside the reconnected field line region in flux transfer events: Theory, Planet. Space Sci., 35, 227-240, 1987.

Farrugia, C. J., R. P. Rijnbeek, M. A. Saunders, D. J. Southwood, D. J. Rodgers, M. F. Smith, C. P. Chaloner, D. S. Hall, P. J. Christiansen, and L. J. C. Woolliscroft, A multi-instrument study of flux transfer event structure, J. Geophys, Res., in press, 1988.

Goertz, C. K. , E. Neilsen, A. Korth, K. -H. Glassmeier, C. Haldoupis, P. Hoeg, and D. Hayward, Ooservations of a possible ground signature of flux transfer events, J. Geophys. Res.., 90, 4069-4078, 1985.

Gombosi, T. I., and T. L. Killeen, Effects of thermospheric motions on the polar wind: A time-dependent numerical study, J. Geophys. Res., 92, 4725-4729, 1987.

Hubert, D., Non-Maxwellian velocity distribution functions and incoherent scattering of radar waves in the auroral ionosphere, J. Atmos. Terr. Phys., 46, 601-612, 1984.

Lockwood, M., Low-energy ion flows into the magnetosphere, Adv. Space Res., 6(3), 63-77, 1986.

Lockwood, M. , M. O. Chandler, J. L. Horwitz, J. H. Waite, Jr., T. E. Moore, and C. R. Chappell, The cleft ion fountain, J. Geophys. Res., 10, 9736-9748, 1985a.

Lockwood M., T. E. Moore, J. H. Waite, Jr., C. R. Chappell, J. L. Horwitz, and R. A. Heelis, The geomagnetic mass spectrometer Mass and energy dispersions of ionospheric ion flows into the magnetosphere, Nature, 316, 612-613, 1985b.

Lockwood, M. , J. H. Waite Jr., T. E. Moore, J. F. E. Johnson, and C. R. Chappell, A new source of suprathermal $\mathrm{O}^{+}$ions near the dayside polar cap boundary, J. Geophys. Res., 90, 4099-4116, 1985c.

Lockwood, M., and T. J. Fuller-Rowell, The modelled occurrence of non-thermal plasma in the ionospheric $F$ region and the possible consequences for ion outflows into the magnetosphere, Geophys. Res. Lett., 14, 371-374, 1987a.

Lockwood, M., and T.J. Fuller-Rowell, Correction to "The modelled occurrence of non-thermal plasma in the ionospheric $F$ region and the possible consequences for ion outflows into the magnetosphere", Geophys. Res. Lett., 14, $581-582,1987 \mathrm{~b}$.

Lockwood, M. , B. J. I. Bromage, R. B. Horne, J.P. St.-Maurice, D. M. Willis, and S. W. H. Cowley, Non-Maxwellian ion velocity distributions observed using EISCAT, Geophys. Res. Lett. , 14, 111-114, 1987.

Lockwood, M., K. Suvanto, J.-P. St.-Maurice, K. Kikuchi, B. J. I. Bromage, D. M. Willis, C. R. Crothers, H. Todd, and S. W. H. Cowley, Scattered power from non-thermal plasmaevidence for coherent echoes? J. Atmos. Terr. Phys., in press, 1988. 
Milne-Thompson, L. M., Theoretical Hydrodynamics, pp229-233, MacMillan, New York, 1955.

Moore, T. E., Acceleration of low-energy magnetospheric plasma, Adv. Space Res., 6(3), 103$112,1986$.

Moore, T. E., M. Lockwood, M. O. Chandler, J. H. Waite, Jr., C. R. Chappell, A. Persoon, and M. Sugiura, Upwelling $\mathrm{O}^{+}$ion source characteristics, J. Geophys. Res., 91, 7019-7031, 1986.

Raman, R. S. V*, J.-P. St.-Maurice, and R. S. B. Ong, Incoherent scattering of radar waves in the auroral ionosphere, J. Geophys. Res.. 86, $4751-4762,1981$.

Rijnbeek, R. P., C. J. Farrugia, D. J. Southwood, M. W. Dunlop, W. C. Mier-Jedrzejowicz, C. P. Chaloner, D. S. Hall, and M. F. Smith, A magnetic boundary signature within flux transfer events, Planet. Space Sci., in press, 1987.

Russell, C. T., and R. C. Elphic, Initial ISEE magnetometer results: Magnetopause observations, Space Sci. Rev., 22, 681, 1978.

Sandholt, P. E., A. Egeland, J. A. Holtet, B. Lybekk, K. Svenes, S. Assheim, and C. S. Deehr, Large- and small-scale dynamics of the polar cusp, J. Geophys. Res.., 90, 4407-4414, 1985.

Sandholt, P. E., C. S. Deehr, A. Egeland, B. Lybekk, R. Viereck, and G. J. Romick, Signatures in the dayside aurora of plasma transfer from the magnetosheath, J. Geophys. Res.., 91, 10,063-10,079, 1986.

Saunders, M. A., C. T. Russell and N. Sckopke, Flux transfer events: Scale size and internal structure, Geophys. Res. Lett., 11, 131-134, 1984.

Schunk, R. W., An updated theory of the polar wind, Adv. Space Res., 6(3), 79-88, 1986.

Sckopke, N., G. Paschmann, G. Haerendel, B. U. 0. Sonnerup, S. J. Bame, T. G. Forbes, E. W. Hones, Jr., and C. T. Russell, Structure of the low-latitude boundary layer, J. Geophys. Res., 86, 2099-2110, 1981.

Scudder, J. D., K. W. Ogilvie, and C. T. Russell, The relationship of flux transfer events to magnetic reconnection, in Magnetic Reconnection in Space and Laboratory Plasmas, Geophys. Monogr. Ser., Vol. 30, edited by E.W. Hones, Jr., p 151, AGU, Washington, D. C., 1984.

Siscoe, G. L., and M. Lockwood, Ionospheric wakes of FTEs (abstract), EOS Trans. AGU, 67 (44), $1162,1986$.
Smith, M. F., D. J. Rodgers, and M. A. Saunders, Ion flows in magnetospheric FTEs, Proceedings of 21st ESLAB Conference, Botkesk $\phi$, Norway, Eur. Apace Agency Spec. Publ., ESA SP-275, 1987.

Sonnerup, B. U. O., on the stress balance in flux transfer events, J. Geophys. Res., 92, 8613, 1987.

Southwood, D. J., Theoretical aspects of ionosphere-magnetosphere-solar wind coupling, Physics of the Ionosphere-Magnetosphere, Adv. Space Res.., 5, 4, 1985.

Southwood, D. J., The ionospheric signature of flux Transfer events, J. Geophys. Res., 92 3207-3213, 1987.

Southwood, D. J., C. J. Farrugia, and M. A. Saunders, What are flux transfer events?, Planet. Space Sci., in press, 1988.

Southwood, D. J., W. A. C. Mier-Jedrzejowicz and C. T. Russel1, The fluxgate magnetometer for the AMPTE UK subsatellite, IEEE Trans. Geosci. Remote Sens.., GE-23, 301-304, 1985.

St.-Maurice, J.-P., and R. W. Schunk, Ion velocity distributions in the high-latitude ionosphere, Rev. Geophys.., 17, 99-134, 1979.

Todd, H., B. J. I. Bromage, S. W. H. Cowley, M. Lockwood, A. P. van Eyken, and D. M. Willis, EISCAT observations of bursts of rapid flow in the high-latitude dayside ionosphere, Geophys. Res. Lett. , 13, 909-912, 1986.

Todd, H., S. W. H. Cowley, A. Etemadi, B. J. I. Bromage, M. Lockwood, and D. M. Willis, Flow in the high latitude ionosphere: Measurements at 15 seconds resolution made using the EISCAT "POLAR" experiment, J. Atmos. Terr. Phys., in press, 1987.

C. J. Farrugia, Blackett Laboratory, Imperial College, London, SW7 2BZ, Fngland. M. Lockwood, Rutherford Appleton Laboratory,

Chilton, Didcot, Oxon, Ox11 0Qx, England. G. L. Siscoe, University of California at Los Angeles, Department of Atmospheric Sciences, Los Angeles, CA 90024 .

M.F. Smith, Southwest Research Institute, San Antonia, TX 78284 .

(Received August 17, 1987,

revised November 23, 1987, accepted December 16, 1987.) 